\title{
Top quark mass measurement with ATLAS
}

\section{Pietro G. CAVALLERI on behalf of the ATLAS collaboration* LPNHE, Paris}

E-mail: pietro.cavalleri@cern.ch

The LHC will be the first top quark factory. $t \bar{t}$ pairs will be produced at such a high rate that statistical error on the top quark mass measurement will soon become negligible.

A precise top quark mass measurement is of fundamental importance because it is one of the fundamental parameter of the Standard Model with a value close to the electroweak symmetry breaking scale. Moreover, during the LHC start-up, it will be a high mass reference candle.

In the ATLAS experiment, the most promising analysis will use tit pairs that decay semileptonically. The different steps of this analysis will be detailed and a quick overview of the other ongoing analyses will be given.

Physics at LHC 2008

29 September - October 4, 2008

Split, Croatia

${ }^{*}$ Speaker. 


\section{Why the top quark mass?}

The DO and CDF experiments at Tevatron have achieved a very precise measurement of the top quark mass with the largest error being from systematics and below 1\% [1]. Nevertheless, an accurate measurement of the heaviest quark is a must for experiments studying physics at the Tera scale for the following reasons:

- During the LHC start-up, it will be a high mass reference candle.

- $\mathrm{tt}$ events, with their complexity, will play a fundamental role in the detector understanding: considering that in the Standard Model the top quark decays always in a W boson plus a $\mathrm{b}$ jet, $\bar{t} \bar{t}$ events will give samples of $b$ jets that will be useful to improve b-tagging algorithms [2]. Moreover, they will help in the Jet Energy Scale (JES) determination [3].

- The top quark mass is a fundamental parameter of the Standard Model. Moreover, it is the only quark that decays before hadronization.

- W boson, top and Higgs masses are related in the Standard Model; a precise measurement of the top mass constrains the Standard Model Higgs boson mass via the relation

$$
M_{W}=4 \sqrt{\frac{\pi^{2} \alpha^{2}}{2 G^{2}}} \frac{1}{\sin \theta_{W}(1-\Delta R)}, \Delta R \propto\left(M_{\text {top }}^{2}, \log M_{H}\right)
$$

The LHC will be a top quark factory: each year, at a center of mass energy of $14 \mathrm{TeV}$ and at an integrated luminosity of $10 \mathrm{fb}^{-1}, 8$ millions of $t \bar{t}$ events will be produced. The statistical error will become negligible and the challenge for the top mass measurement will be to reduce the systematic error.

\section{Analysis channels}

In the Standard Model, the top quark decays in a $\mathrm{W}$ boson and a b quark with a branching ratio close to 1 . This means that the $\mathrm{W}$ decay determines the analysis channel.

All Jets channel The two electro-weak bosons decay hadronically. This channel has the highest statistics with a branching ratio of $44 \%$ (Figure 1) and gives the possibility to reconstruct the whole event, but it suffers from a huge QCD background and the corresponding combinatorial background.

Dileptons channel The two W bosons decay leptonically. It might be considered as complementary to the All Jets channel: it has very low branching ratio and it is impossible to perform a direct measurement of the invariant mass because of the two undetectable neutrinos. However, the clean signature resulting from the two high $p_{T}$ leptons and the high missing transverse energy, make it easier to detect and the background is low.

Lepton plus jets channel This channel is the best compromise between a large branching ratio and a rather low background: the high $p_{T}$ lepton provides an efficient trigger for the signal while the hadronic $\mathrm{W}$ and the associated $\mathrm{b}$ jet allow the top invariant mass reconstruction (Figure 1). 

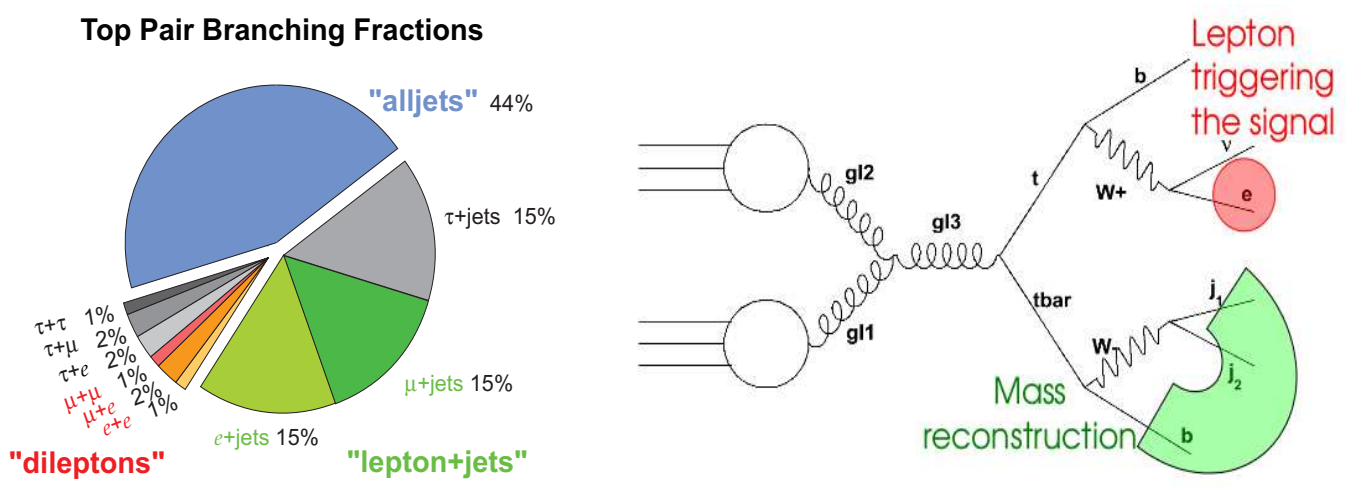

Figure 1: Left: $\bar{t} \bar{t}$ branching ratios. Right: Feynman diagram for $t \bar{t} \rightarrow b e v, b j j$. The high $p_{T}$ lepton is used to trigger the signal, jets from the hadronically decaying $W$ are used for the top mass reconstruction.

\section{Lepton plus jets channel.}

One of the most promising analysis is performed on a clean sample of top events selected requiring two b-tagged jets [3].

Event Selection The main backgrounds after these selection cuts are the $\mathrm{W}+\mathrm{b} \overline{\mathrm{b}}$ and $\mathrm{W}+\mathrm{c} \overline{\mathrm{c}}$ production with the $\mathrm{W}$ boson decaying leptonically, the $\mathrm{Z}+$ jets events with $\mathrm{Z} \rightarrow 1$, single top events and fully hadronic and fully leptonic $t \bar{t}$ events.

The cuts applied to increase the ratio Signal over Background are the following:

- only one isolated lepton with a transverse momentum $p_{T}$ greater than 20 (25) $\mathrm{GeV}$ for muons (electrons). The isolation request rejects all the leptonic b-decays in the fully hadronic $\mathfrak{t} \bar{t}$ channel.

- A missing transverse energy $\left(M E_{T}\right)$ greater that $20 \mathrm{GeV}$, due to the undetected neutrino in the signal events, reduces QCD background.

- At least 4 jets with $p_{T}>40 \mathrm{GeV}$ to reduce the fully leptonic and QCD backgrounds.

The impact of these cuts on the signal and on the backgrounds are shown in Table 1.

Hadronic W mass reconstruction The Jet Energy Scale, defined as the ratio between the measured clustered jet energy and the quark energy, depends on the jet energy. In this analysis an in situ jet rescaling has been implemented, giving a jet energy dependent correction. This event-by-event rescaling is performed by constraining the jet pairs to the known $\mathrm{W}$ mass by a $\chi^{2}$ minimization.

$$
\chi^{2}=\frac{\left(M_{j j}\left(\alpha_{E_{j 1}}, \alpha E_{j 2}\right)-M_{W}^{P D G}\right)^{2}}{\Gamma_{W}^{P D G}}+\frac{E_{j 1}\left(1-\alpha_{E_{j 1}}\right)^{2}}{\sigma_{1}^{2}}+\frac{E_{j 2}\left(1-\alpha_{E_{j 2}}\right)^{2}}{\sigma_{2}^{2}}
$$

The jet pair with the smallest $\chi^{2}$ is kept as the hadronic $\mathrm{W}$ candidate (Figure 2). This $\chi^{2}$ minimization procedure allows also the jets energy to vary according to the resolution $\sigma_{i}$ expected for that energy. 

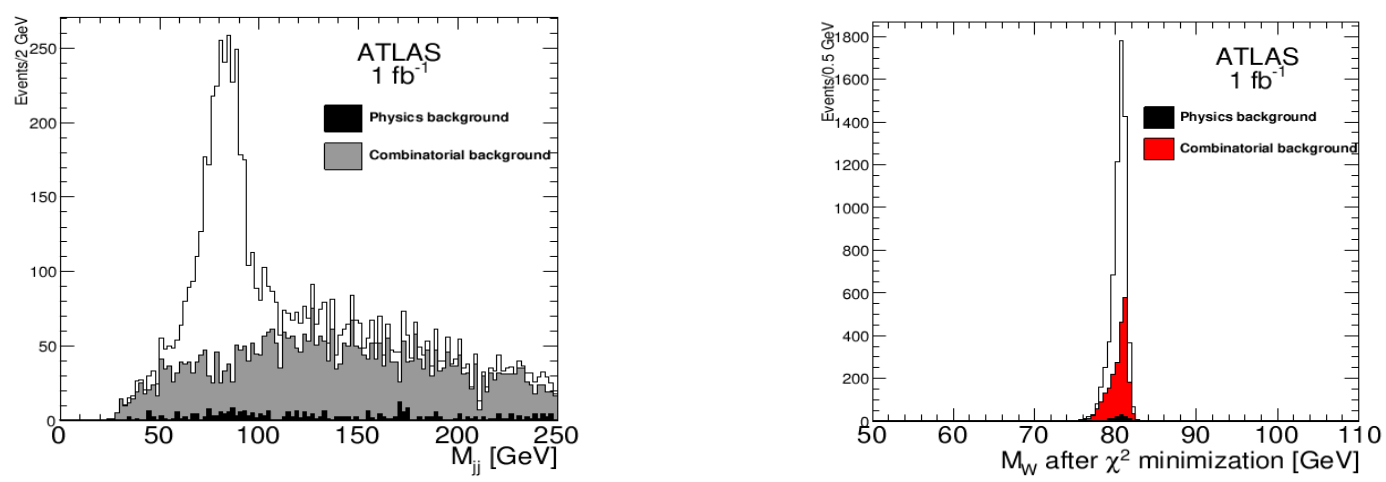

Figure 2: $\mathrm{W}$ mass reconstruction in lepton plus jets channel. Left: $\mathrm{W}$ invariant mass before $\chi^{2}$ minimization. The combinatorial background arises from an incorrect combination of jets used to reconstruct the $W$ boson. Right: W invariant mass after in situ rescaling done by $\chi^{2}$ minimization
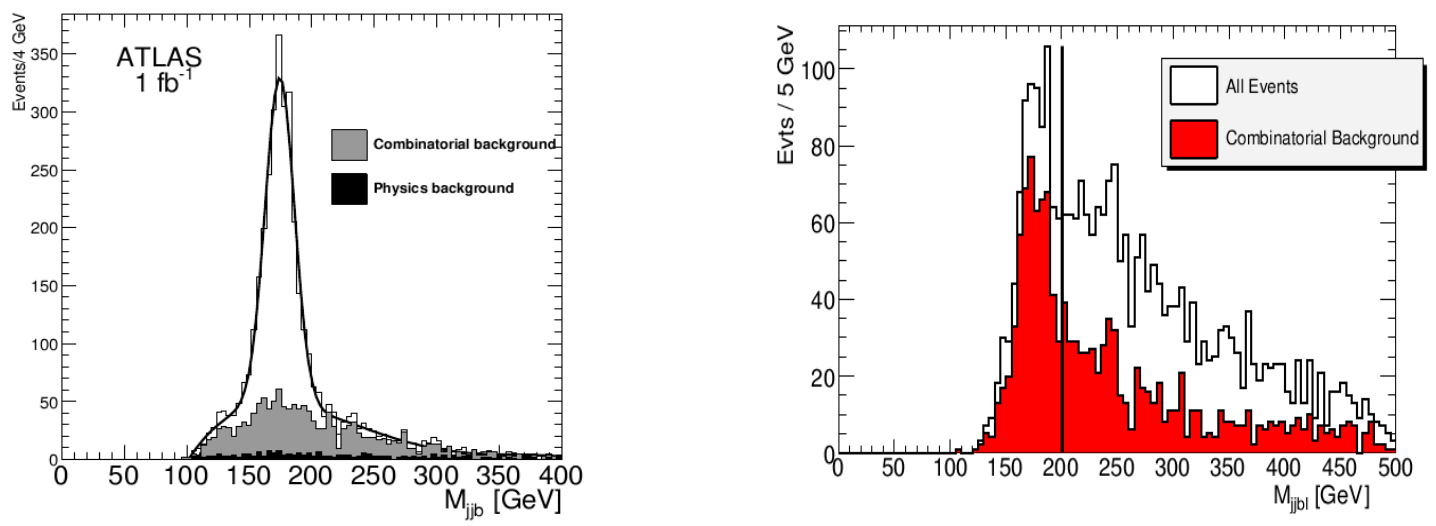

Figure 3: Left: Gaussian plus cubic fit for the Top Quark invariant mass. Right: invariant mass of the hadronic $\mathrm{W}$ and leptonic b-jet.

Top quark mass reconstruction and measurement Once the hadronic W boson has been reconstructed, the associated b-jet has to be chosen. Three methods have been tested [3] leading to similar results. The method chosen, that maximizes the purity of the signal, is to use the b-jet closest to the hadronic W. The value given by the fit on the invariant mass (Figure 3 left) is

$$
M_{\text {top }}=175.0 \pm 0.2 \mathrm{GeV}, \quad \Gamma_{\text {top }}=11.6 \pm 0.2 \mathrm{GeV}
$$

for a Monte Carlo sample generated with $\mathrm{m}_{\text {top }}$ at $175 \mathrm{GeV} / \mathrm{c}^{2}$. Additional cuts have been applied in order to improve the final top purity [3]. Among them, the most efficient ones are the following:

- The invariant mass of the hadronic $\mathrm{W}$ boson and leptonic b-jet has to be greater than 200 $\mathrm{GeV}$ (Figure 3 right).

- The invariant mass of the isolated lepton and leptonic b-jet has to be smaller than $160 \mathrm{GeV}$.

The systematic uncertainties are listed in Table 1. The Jet Energy Scale (JES) is the main source of systematic uncertainty. The JES for lights jets is strongly reduced thanks to the in situ 


\begin{tabular}{|c|c|c|c|c|c|c|}
\hline \multirow[t]{2}{*}{ Process } & Number & 1 isol. lep & $\geq 4$ jets & 2 b-jets & \multirow[b]{2}{*}{ Systematics } & \\
\hline & of events & $p_{T}>20, \mathrm{E}_{\mathrm{T}}>20$ & $p_{T}>40$ & $p_{T}>40$ & & \\
\hline Signal & 313200 & 132380 & 43370 & 15780 & Light $J E S$ & $0.2 \mathrm{GeV} / \%$ \\
\hline$\overline{\mathrm{W}+\text { jets }}$ & $9.5 \times 10^{5}$ & 154100 & 9450 & 200 & b JES & $0.7 \mathrm{GeV} / \%$ \\
\hline Z+jets & $1.2 \times 10^{6}$ & 180000 & 570 & 20 & ISR/FSR & $\simeq 0.3 \mathrm{GeV}$ \\
\hline$t \bar{t} \rightarrow$ alljets & 466480 & 1020 & 560 & 160 & b quark fragm. & $\leq 0.1 \mathrm{GeV}$ \\
\hline$t \bar{t} \rightarrow$ dilep & 52500 & 16470 & 2050 & 720 & Background & negligible \\
\hline single top & 81500 & 24400 & 1230 & 330 & Method & 0.1 to $0.2 \mathrm{GeV}$ \\
\hline
\end{tabular}

Table 1: Left: cuts impact on signal and background. Right: systematic errors. The dominant is the b JES.

rescaling. For an error on the Jet Energy Scale of the order of $1 \%(5 \%)$ the systematic error is $\sim 1$ (3.5) GeV.

\section{Other ongoing analysis}

Many other analyses to measure the top quark mass are performed in ATLAS: they are important cross checks for the analysis described above. Among the ongoing analyses on the dileptons channel there are:

Matrix Element method A calculation of a per event probability density for signal and background as a function of the top quark mass is performed. This probability density consists in a convolution of the Matrix Element of the process with the detector resolution functions, integrating over the unmeasured quantities of the phases space (the neutrinos momenta).

Decay length method The sensitivity of the B decays length on the top mass is exploited: the goal is to correlate the mean transverse decay of the B to the top mass. This method does not depend on the Jet Energy Scale which is the main source of systematic error of the analyses described above.

\section{Conclusion}

The top quark mass measurement remains of fundamental importance for physics at the Tera scale. At ATLAS the most promising analysis on the lepton plus jets channel allows to measure it with a statistical error of $0.2 \mathrm{GeV} / \mathrm{c}^{2}$. The systematic error is $1(3.5) \mathrm{GeV} / \mathrm{c}^{2}$ with a Jet Energy Scale of $1 \%(5 \%)$ for an integrated luminosity of $1 \mathrm{fb}^{-1}$. The top mass measurement is also performed in other channels with different source of systematics errors, giving important cross-checks.

\section{References}

[1] Combination of CDF and D0 Results on the Mass of the Top Quark" The Tevatron Electroweak Working Group, for the CDF, D0 Collaborations, http://arxiv.org/abs/0808.1089v1

[2] ATLAS collaboration, "b-Tagging",this volume.

[3] Atlas Collaboration, Expected Performance of the ATLAS Experiment, Detector, Trigger and Physics, CERN-OPEN-2008-020, Geneva, 2008, to appear. 